SLAC-PUB-6661

\title{
Beta decay of hyperons in a relativistic quark model
}

\author{
Felix Schlumpf \\ Stanford Linear Accelerator Center \\ Stanford University, Stanford, California 94309
}

(June 18, 2021)

\begin{abstract}
A relativistic constituent quark model is used to calculate the semileptonic beta decay of nucleons and hyperons. The parameters of the model, namely, the constituent quark mass and the confinement scale, are fixed by a previous calculation of the magnetic moments of the baryon octet within the same model. We discuss the momentum dependence of the form factors, possible configuration mixing and $\mathrm{SU}(3)$ symmetry breaking. We conclude that the relativistic constituent quark model is a good framework to analyze electroweak properties of the baryons.
\end{abstract}

PACS numbers: 14.20.Jn, 12.39.Ki, 13.30.Ce 


\section{INTRODUCTION}

In this paper we consider the application of the relativistic constituent quark model to the semileptonic hyperon decay. We compare our result with the new data from the particle data group [1]. The predictive power of a relativistic constituent quark model formulated on the light-front was recently investigated in Ref. [2]. It provides a simple model wherein we have overall an excellent and consistent picture of the magnetic moments and of the semileptonic decays of the baryon octet. This paper extends the analysis of the semileptonic beta decays and addresses specific questions for the hyperon beta decay.

The effect of configuration mixing has recently been studied [3] in the context of deep inelastic scattering. We show below that such a configuration mixing is not favored for hyperon decays.

Our quark model provides a unique scheme for calculating the momentum dependence of the form factors. Although it is generally small, a change of the dipole masses $M_{V}$ or $M_{A}$ by $\pm 0.15 \mathrm{GeV}$ in the case $\Sigma^{-} \rightarrow n e \nu$ causes a relative change of $g_{1} / f_{1}$ of $\pm 2 \%$. Ignoring the momentum dependence altogether would shift $g_{1} / f_{1}$ by $17 \%$.

The SU(3) symmetry breaking can also be studied in our model. It plays a major role in the determination of the Kobayashi-Maskawa-Cabibbo matrix element $V_{u s}$ from baryon decay.

The parameters of the model are the constituent quark mass $m$ and the scale parameter $\beta$, which is a measure for the size of the baryon. All parameters have been determined and fixed in Ref. [2]. The results reported in this paper are independent of the wave function assumed in the calculation. It has been shown in Ref. [4 that relations between observables at zero momentum transfer are independent of the wave function, and Ref. [5] shows that this independence holds up to $1 \mathrm{GeV}^{2}$ for the baryons.

This article is organized as follows. Section $\llbracket$ describes the basics of hyperon semileptonic decay. In Sec. [II we give a brief summary of our model as described in Ref. [2] with the explicit expressions for the beta decay. The numerical results are presented in Sec. IV, and are compared with experiment, other calculations, and some extensions of the model. We summarize our investigation in a concluding Sec. $\nabla$.

\section{HYPERON SEMILEPTONIC DECAY}

In the low energy limit the standard model for semileptonic weak decays reduces to an effective current-current interaction Hamiltonian

$$
H_{\text {int }}=\frac{G}{\sqrt{2}} J_{\mu} L^{\mu}+\text { h.c. , }
$$

where $G \simeq 10^{-5} / M_{p}^{2}$ is the weak coupling constant,

$$
L^{\mu}=\bar{\psi}_{e} \gamma^{\mu}\left(1-\gamma_{5}\right) \psi_{\nu}+\bar{\psi}_{\mu} \gamma^{\mu}\left(1-\gamma_{5}\right) \psi_{\nu}
$$

is the lepton current, and

$$
\begin{aligned}
J_{\mu} & =V_{\mu}-A_{\mu}, \\
V_{\mu} & =V_{u d} \bar{u} \gamma_{\mu} d+V_{u s} \bar{u} \gamma_{\mu} s, \\
A_{\mu} & =V_{u d} \bar{u} \gamma_{\mu} \gamma_{5} d+V_{u s} \bar{u} \gamma_{\mu} \gamma_{5} s,
\end{aligned}
$$


is the hadronic current, and $V_{u d}, V_{u s}$ are the elements of the Kobayashi-Maskawa mixing matrix. The $\tau$-lepton current cannot contribute since $m_{\tau}$ is much too large.

The matrix elements of the hadronic current between spin- $\frac{1}{2}$ states are

$$
\begin{gathered}
\left\langle B^{\prime}, p^{\prime}\left|V^{\mu}\right| B, p\right\rangle=V_{q q^{\prime}} \bar{u}\left(p^{\prime}\right)\left[f_{1}\left(K^{2}\right) \gamma^{\mu}-\frac{f_{2}\left(K^{2}\right)}{M_{i}} i \sigma^{\mu \nu} K_{\nu}+\frac{f_{3}\left(K^{2}\right)}{M_{i}} K^{\mu}\right] u(p), \\
\left\langle B^{\prime}, p^{\prime}\left|A^{\mu}\right| B, p\right\rangle=V_{q q^{\prime}} \bar{u}\left(p^{\prime}\right)\left[g_{1}\left(K^{2}\right) \gamma^{\mu}-\frac{g_{2}\left(K^{2}\right)}{M_{i}} i \sigma^{\mu \nu} K_{\nu}+\frac{g_{3}\left(K^{2}\right)}{M_{i}} K^{\mu}\right] \gamma_{5} u(p),
\end{gathered}
$$

where $K=p-p^{\prime}$ and $M_{i}$ is the mass of the initial baryon. The quantities $f_{1}$ and $g_{1}$ are the vector and axial-vector form factors, $f_{2}$ and $g_{2}$ are the weak magnetism and electric form factors and $f_{3}$ and $g_{3}$ are the induced scalar and pseudoscalar form factors, respectively. Time invariance implies real form factors. We do not calculate $f_{3}$ and $g_{3}$ since we put $K^{+}=0$ and their dependence on the decay spectra is of the order

$$
\left(\frac{m_{l}}{M_{i}}\right)^{2} \ll 1
$$

where $m_{l}$ is the mass of the final charged lepton. The other form factors are

$$
\begin{aligned}
f_{1} & =\left\langle B^{\prime}, \uparrow\left|V^{+}\right| B, \uparrow\right\rangle, \\
K_{\perp} f_{2} & =M_{i}\left\langle B^{\prime}, \uparrow\left|V^{+}\right| B, \downarrow\right\rangle, \\
g_{1} & =\left\langle B^{\prime}, \uparrow\left|A^{+}\right| B, \uparrow\right\rangle, \\
K_{\perp} g_{2} & =-M_{i}\left\langle B^{\prime}, \uparrow\left|A^{+}\right| B, \downarrow\right\rangle .
\end{aligned}
$$

What is usually measured is the total decay rate $\Gamma$, the electron-neutrino correlation $\alpha_{e \nu}$ and the electron $\alpha_{e}$, neutrino $\alpha_{\nu}$ and final baryon $\alpha_{B}$ asymmetries. The $e-\nu$ correlation is defined as

$$
\alpha_{e \nu}=2 \frac{N\left(\Theta_{e \nu}<\frac{1}{2} \pi\right)-N\left(\Theta_{e \nu}>\frac{1}{2} \pi\right)}{N\left(\Theta_{e \nu}<\frac{1}{2} \pi\right)+N\left(\Theta_{e \nu}>\frac{1}{2} \pi\right)},
$$

where $N\left(\Theta_{e \nu}<\frac{1}{2} \pi\right)$ is the number of $e-\nu$ pairs that form an angle $\Theta_{e \nu}$ smaller than $90^{\circ}$. The correlations $\alpha_{e}, \alpha_{\nu}$ and $\alpha_{B}$ are defined analogously with $\Theta_{e}, \Theta_{\nu}$ and $\Theta_{B}$ now being the angles between the $e, \nu, B$ directions and the polarization of the initial baryon.

Ignoring the lepton-mass one can calculate expressions for the measured quantities. Expressions for $\Gamma, \alpha_{e \nu}, \alpha_{e}, \alpha_{\nu}$ and $\alpha_{B}$ are given in Ref. [6]. For the decay rate $\Gamma$ we have for instance:

$$
\begin{aligned}
\Gamma= & G^{2} \frac{\Delta M^{5}|V|^{2}}{60 \pi^{3}}\left[\left(1-\frac{3}{2} \beta+\frac{6}{7} \beta^{2}\right) f_{1}^{2}+\frac{4}{7} \beta^{2} f_{2}^{2}+\left(3-\frac{9}{2} \beta+\frac{12}{7} \beta^{2}\right) g_{1}^{2}\right. \\
& \left.+\frac{12}{7} \beta^{2} g_{2}^{2}+\frac{6}{7} \beta^{2} f_{1} f_{2}+\left(-4 \beta+6 \beta^{2}\right) g_{1} g_{2}+\frac{4}{7} \beta^{2}\left(f_{1} \lambda_{f}+5 g_{1} \lambda_{g}\right)\right],
\end{aligned}
$$

where $\beta$ is defined as $\beta=\left(M_{i}-M_{f}\right) / M_{i}$, and $\Delta M=M_{i}-M_{f}, M_{i}, M_{f}$ being the masses of the initial and final baryon, respectively. The $K^{2}$ dependence of $f_{2}$ and $g_{2}$ is ignored and $f_{1}$ and $g_{1}$ are expanded as 


$$
f_{1}\left(K^{2}\right)=f_{1}(0)+\frac{K^{2}}{M_{i}^{2}} \lambda_{f}, \quad g_{1}\left(K^{2}\right)=g_{1}(0)+\frac{K^{2}}{M_{i}^{2}} \lambda_{g}
$$

We get the corresponding expression for the dipole parameterization $f\left(K^{2}\right)=\left(1-K^{2} / M^{2}\right)^{-2}$ by putting

$$
\lambda_{f}=2 M_{i}^{2} f_{1} / M_{V}^{2}, \quad \lambda_{g}=2 M_{i}^{2} g_{1} / M_{A}^{2}
$$

These quantities are corrected by the nonvanishing lepton mass and radiative corrections [6].

\section{THE FORM FACTORS IN A RELATIVISTIC CONSTITUENT QUARK MODEL}

The constituent quark model described in Ref. [2] provides a framework for representing the general structure of the three-quark wave function for baryons. The model is formulated on the light-front, which is specified by the invariant hypersurface $x^{+}=x^{0}+x^{3}=0$. The wave function is constructed as the product of a momentum wave function, which is spherically symmetric and invariant under permutations, and a spin-isospin wave function, which is uniquely determined by $\mathrm{SU}(6)$ symmetry requirements. A Wigner (Melosh) rotation 99 is applied to the spinors, so that the wave function of the proton is an eigenfunction of $J^{2}$ and $J_{z}$ in its rest frame [10]. To represent the range of uncertainty in the possible form of the momentum wave function, harmonic oscillator and a pole-type wave function have been chosen in Refs. [2, 14.5]. Surprisingly, it has been found that observables at zero momentum transfer are independent of the wave function chosen [⿴囗十), and form factors do not differ up to $1 \mathrm{GeV}^{2}$ [5] for a wide range of wave functions. Since the momentum transfer involved in hyperon beta decays is much smaller than $1 \mathrm{GeV}^{2}$ it is representative to use one special wave function. The form factors in Eq. 2.7 are calculated as shown in Ref. [2]. In contrast to Ref. [2], we do not assume additional structure of the constituent quarks, and we choose

symmetric wave functions. These simplifications reduce the number of free parameters to two masses $\left(m_{u / d}, m_{s}\right)$ and three scale parameters $\left(\beta_{N}, \beta_{\Sigma / \Lambda}, \beta_{\Xi}\right)$.

For $K^{2}=0$ we have for $\Delta S=0$ transitions

$$
\begin{aligned}
f_{1} & =A\left(f_{1}\right), \\
f_{2} & =\frac{N_{c}}{(2 \pi)^{6}} \int d^{3} q d^{3} Q|\Phi|^{2} A\left(f_{2}\right), \\
g_{1} & =A\left(g_{1}\right) \frac{N_{c}}{(2 \pi)^{6}} \int d^{3} q d^{3} Q|\Phi|^{2} \frac{b^{2}-Q_{\perp}^{2}}{b^{2}+Q_{\perp}^{2}}, \\
g_{2} & \simeq 0,
\end{aligned}
$$

with $A$ s given in Table 1 . The values $A\left(f_{1}\right)$ and $A\left(g_{1}\right)$ are the values in the nonrelativistic quark model. The factors $A_{1}, A_{2}$, and $A_{3}$ are given in Eq. (3.6) of Ref. [11.

The $\Delta S=1$ transitions for $K^{2}=0$ are 


$$
\begin{aligned}
& f_{1}=\frac{N_{c}}{(2 \pi)^{6}} \int d^{3} q d^{3} Q\left(\frac{E_{3}^{\prime} E_{12}^{\prime} M}{E_{3} E_{12} M^{\prime}}\right)^{1 / 2} \frac{\Phi^{\dagger}\left(M^{\prime}\right) \Phi(M) B\left(f_{1}\right)}{\left(a^{\prime 2}+Q_{\perp}^{2}\right)\left(a^{2}+Q_{\perp}^{2}\right) \sqrt{b^{\prime 2}+Q_{\perp}^{2}} \sqrt{b^{2}+Q_{\perp}^{2}}} \\
& g_{1}=\frac{N_{c}}{(2 \pi)^{6}} \int d^{3} q d^{3} Q\left(\frac{E_{3}^{\prime} E_{12}^{\prime} M}{E_{3} E_{12} M^{\prime}}\right)^{1 / 2} \frac{\Phi^{\dagger}\left(M^{\prime}\right) \Phi(M) B\left(g_{1}\right)}{\left(a^{\prime 2}+Q_{\perp}^{2}\right)\left(a^{2}+Q_{\perp}^{2}\right) \sqrt{b^{\prime 2}+Q_{\perp}^{2}} \sqrt{b^{2}+Q_{\perp}^{2}}} \\
& B\left(f_{1}\right)= B_{1}\left(a^{\prime} a+Q_{\perp}^{2}\right)^{2}\left(b^{\prime} b+Q_{\perp}^{2}\right) \\
&+B_{2}\left(a^{\prime}-a\right)^{2} Q_{\perp}^{2}\left(b^{\prime} b+Q_{\perp}^{2}\right) \frac{\left(c d-q_{\perp}^{2}\right)^{2}}{\left(c^{2}+q_{\perp}^{2}\right)\left(d^{2}+q_{\perp}^{2}\right)} \\
&+B_{3}\left(a^{\prime}-a\right)\left(b^{\prime}-b\right) Q_{\perp}^{2}\left(a^{\prime} a+Q_{\perp}^{2}\right)\left(\frac{c^{2}}{c^{2}+q_{\perp}^{2}}+\frac{d^{2}}{d^{2}+q_{\perp}^{2}}\right) \\
& B\left(g_{1}\right)= B_{4}\left(b^{\prime} b-Q_{\perp}^{2}\right)\left[\left(a^{\prime} a+Q_{\perp}^{2}\right)^{2}+\left(a^{\prime}-a\right)^{2} Q_{\perp}^{2} \frac{\left(c d-q_{\perp}^{2}\right)^{2}}{\left(c^{2}+q_{\perp}^{2}\right)\left(d^{2}+q_{\perp}^{2}\right)}\right] \\
&+B_{5}\left(a^{\prime}-a\right)^{2} Q_{\perp}^{2}\left(b^{\prime} b-Q_{\perp}^{2}\right) \frac{c d q_{\perp}^{2}}{\left(c^{2}+q_{\perp}^{2}\right)\left(d^{2}+q_{\perp}^{2}\right)} \\
&+B_{6}\left(a^{\prime}-a\right) Q_{\perp}^{2}\left(b^{\prime}+b\right)\left(a^{\prime} a+Q_{\perp}^{2}\right)\left(\frac{c^{2}}{c^{2}+q_{\perp}^{2}}+\frac{d^{2}}{d^{2}+q_{\perp}^{2}}\right)
\end{aligned}
$$

The $B_{i}$ for the different decays are given in Table $\Pi$. The quantities $a, b, c, d, q_{\perp}, Q_{\perp}, E_{3}, E_{12}$, and $M$ are defined in Eqs. (2.1), (2.3), (2.4) and (3.8) of Ref. [2].

Eqs. (3.2) and (3.3) confirm the Ademollo-Gatto theorem [12]. Since $\left(a^{\prime}-a\right) \sim \Delta m$ and $\left(b^{\prime}-b\right) \sim \Delta m$ the symmetry breaking for $f_{1}$ is of the order $(\Delta m)^{2}$ whereas it is of the order $\Delta m$ for $g_{1}$ owing to the term containing $B_{6}$. In addition to Ademollo-Gatto we see that the symmetry breaking for $g_{1}(\Lambda \rightarrow p)$ is also of second order.

The full formulae for $K^{2} \leq 0$ are longer than the ones for $K^{2}=0$; they are given in Ref. [13].

\section{NUMERICAL RESULTS}

The form factors can be determined by the generalization of Eqs. (3.1) and (3.2). With the parameterization of the form factor $f\left(K^{2}\right)$ :

$$
f\left(K^{2}\right) \simeq \frac{f(0)}{1-K^{2} / \Lambda_{1}^{2}+K^{4} / \Lambda_{2}^{4}},
$$

we get the result shown in Tables III and [V together with the rates, angular correlation and asymmetries. The parameters $\Lambda_{n}$ are determined by the calculation of the appropriate derivatives of $f\left(K^{2}\right)$ at $K^{2}=0$. The rates have been corrected taking into account the nonvanishing lepton mass and radiative corrections.

In this paper, we use the parameter set 2 of Ref [2]. The values for the constituent quark masses and the confinement scales are 


$$
\begin{aligned}
m_{u}=m_{d} & =0.267 \mathrm{GeV}, \\
m_{s} & =0.40 \mathrm{GeV}, \\
\beta_{N} & =0.56 \mathrm{GeV}, \\
\beta_{\Sigma}=\beta_{\Lambda} & =0.60 \mathrm{GeV}, \\
\beta_{\Xi} & =0.62 \mathrm{GeV} .
\end{aligned}
$$

These parameters also give good results for the magnetic moments of the baryon octet [2].

\section{A. The rates, $f_{1}(0)$, and $g_{1}(0)$}

The largest discrepancy between theory and experiments comes from the rates and $g_{1} / f_{1}$ for the processes $\Lambda \rightarrow p e^{-} \bar{\nu}_{e}$ and $\Sigma^{-} \rightarrow n e^{-} \bar{\nu}_{e}$. By changing the axial couplings of the quarks, i.e. $g_{1 u s} \simeq 0.9$, we could improve the rates of both reactions, but the ratios $g_{1} / f_{1}$ clearly force us to use $g_{1 u s}=1$. Another modification could be the $\Lambda-\Sigma^{0}$-mixing, which was considered in Ref. [14]. Let us write

$$
\begin{aligned}
& \Lambda_{\text {phys }}=\Lambda \cos \phi+\Sigma^{0} \sin \phi, \\
& \Sigma_{\text {phys }}^{0}=-\Lambda \sin \phi+\Sigma^{0} \cos \phi .
\end{aligned}
$$

A reasonable value for the mixing angle is $\phi=-0.015$ [14] which lies within one standard deviation of experiment [15]. The decay rate and the ratio $g_{1} / f_{1}$ are only modified by some percent with this mixing angle, not helping the disagreement between theory and experiment.

This inconsistency of our values is a general feature of quark models with a SU(6) flavorspin symmetry [16]. The ratio $g_{1} / f_{1}$ can generally be written as

$$
\frac{g_{1}}{f_{1}}=\rho \eta\left(\frac{g_{1}}{f_{1}}\right)_{\text {non-rel }},
$$

where $\left(g_{1} / f_{1}\right)_{\text {non-rel }}$ is the non-relativistic value. The quantity $\rho$ is a relativistic suppression factor due to the "small " components in the quark spinors (in the bag-model) or due to the Melosh-transformation (in our model). The quantity $\eta$ is an enhancing factor due to $\mathrm{SU}(3)$ symmetry breaking in $\Delta S=1$ transitions. From Tables [II] and [V] we see that $\rho \simeq 0.73-0.76$ [4] depending on the strangeness content of the wave functions and $\eta \simeq 1.11$. This simple estimate shows that every quark model is a priori constrained to

$$
\frac{g_{1} / f_{1}\left(\Lambda \rightarrow p e^{-} \bar{\nu}_{e}\right)}{g_{1} / f_{1}\left(\Sigma^{-} \rightarrow n e^{-} \bar{\nu}_{e}\right)}=-3
$$

in contrast to the experimental value $-2.11 \pm 0.15$ for $g_{2}=0$. This puzzle was pointed out independently by Lipkin [17] and the author [13]. For $g_{2} \neq 0$ it is measured that [18]

$$
\left|\frac{g_{1}}{f_{1}}\right|_{\Lambda p}=0.715+0.28 \frac{g_{2}}{f_{1}},
$$

and [19]

$$
\left|\frac{g_{1}}{f_{1}}-0.237 \frac{g_{2}}{f_{1}}\right|_{\Sigma^{-} n}=0.34 \pm 0.017,
$$

which will bring the data closer to -3 , but in our model $g_{2} / g_{1} \simeq 0.025$ which is much too small to remove the discrepancy. 


\section{B. Configuration mixing}

In this Section we investigate the effect caused by configuration mixing suggested by spectroscopy. The analysis of the $\Delta$-nucleon mass splitting suggests [20,21]:

$$
\mid \text { Baryon }\rangle=\mathrm{A}\left[56,0^{+}\right]+\mathrm{B}\left[56,0^{+}\right]^{*}+\mathrm{C}\left[70,0^{+}\right]
$$

in the notation $\left[\mathrm{SU}(6), \mathrm{L}^{p}\right]$, where $\mathrm{A}^{2}+\mathrm{B}^{2}+\mathrm{C}^{2}=1, \mathrm{~L}$ denotes the angular momentum, and $p$ is the parity of the nucleon. The values for $\mathrm{A}, \mathrm{B}, \mathrm{C}$ are listed in Table $\mathrm{V}$ for different references.

Unfortunately, the mixing configuration does not improve the fit, it is even worse for the crucial ratio in Eq. (4.4). A rough estimate gives

$$
\frac{g_{1} / f_{1}\left(\Lambda \rightarrow p e^{-} \bar{\nu}_{e}\right)}{g_{1} / f_{1}\left(\Sigma^{-} \rightarrow n e^{-} \bar{\nu}_{e}\right)} \simeq-3\left(1+\frac{8}{3} \mathrm{C}^{2}\right)=-3.5 \pm 0.1
$$

to be compared with the value -3 for no mixing, and the experimental data $-2.11 \pm 0.15$. Other values like the ratio $\mu(p) / \mu(n)$ also get worse with the configuration mixing suggested in Eq. 4.7. A configuration mixing has recently been suggested in the context of deep inelastic scattering [3]. Equation 4.8 shows that such a possibility is not favored for hyperon decays.

\section{The form factors $f_{2}(0)$ and $g_{2}(0)$}

Our model agrees with the conserved vector current (CVC) hypothesis. The deviations have the same origin as the too small neutron magnetic moment [2] since $f_{2}$ and the magnetic moments have similar analytic forms. The experimental situation is not yet clear, some experiments favor [19] and some disfavor [22] CVC.

For $\Delta S=1$ transitions the prediction of $g_{2} / g_{1}$ for nonrelativistic quark models is $\sim 0.37$ and for the bag model $\sim 0.15$ [23]. Our model gives also a constant value

$$
\left(\frac{g_{2}}{g_{1}}\right)_{\Delta S=1} \simeq 0.025 .
$$

For $\Delta S=0$ transitions we get

$$
\left(\frac{g_{2}}{g_{1}}\right)_{\Delta S=0} \simeq 0.0033,
$$

if we put $m_{d}-m_{u}=7 \mathrm{MeV}$. This confirms the viewpoint of the PDG [1] which fixes $g_{2}=0$. Experiments also find a vanishing or small $g_{2}$ [6].

With CVC and the absence of $g_{2}$ we reach the same conclusion that was reached in nuclear physics. 


\section{D. $K^{2}$-dependence of the form factors}

Tables $[I I$ and $[\mathrm{IV}$ suggest that the form factor of Eq. (4.1) can be approximated by the dipole form

$$
f\left(K^{2}\right) \simeq \frac{f(0)}{\left(1-K^{2} / \Lambda_{2}^{2}\right)^{2}} .
$$

The axial vector form factor $g_{1}$ for the neutron decay gives a value $M_{A}=\Lambda_{2}=1.04 \mathrm{GeV}$ compared to the experimental value $M_{A}=(1.00 \pm 0.04) \mathrm{GeV}$ [24,25].

If we take the dipole Ansatz we can compare our values for $M_{V}$ and $M_{A}$ with the results of other work (see Table $\nabla \mathbb{1})$.

The contribution of $M_{V}$ and $M_{A}$ to the rate and to $x=g_{1} / f_{1}$ to first order is

$$
\begin{aligned}
\frac{\Delta \Gamma}{\Gamma} & =\frac{8}{7} \frac{\beta^{2} M^{2}}{\left(1+3 x^{2}\right)}\left(\frac{1}{M_{V}^{2}}+\frac{5 x^{2}}{M_{A}^{2}}\right) \\
\frac{\Delta x^{2}}{x^{2}} & =-\frac{8}{7} \beta^{2} M^{2}\left[\frac{\left(1-\alpha_{e \nu}\right) \alpha_{e \nu}}{M_{V}^{2}}+\frac{6+5 \alpha_{e \nu}}{M_{A}^{2}}\right]
\end{aligned}
$$

which shows that our parameters give for the decay $\Sigma^{-} \rightarrow n e^{-} \bar{\nu}_{e}$ a $0.3 \%$ larger rate and a $4 \%$ smaller $g_{1} / f_{1}$ than with the parameters of Gaillard et al. that are often used for the experimental analysis. Although this does not explain the inconsistency of the data with our calculation, it shows that future high-statistics experiments should pay more attention to $M_{V}$ and $M_{A}$ in analyzing $g_{1} / f_{1}$.

\section{E. SU(3) symmetry breaking}

There are some questions concerning flavor SU(3) breaking in semileptonic weak hyperon decays [26 28]. In a recent, careful analysis Ref. [29] shows that there is both consistency and evidence for SU(3) breaking. The SU(3) symmetry breaking for $f_{1}$ and $g_{1}$ within our model is given in Tables VII and VIII, respectively. It originates from the mass difference $\Delta m=m_{s}-m_{u / d}$; and it is included to all orders of $\Delta m$ in our approach. The values in the present model are similar to the bag model calculation of Ref. [23]. Note that the center of mass corrections are already included in our formalism. Reference [30] suggests that $f_{1} / f_{1}^{S U(3)}>1$ to reconcile the value for $V_{u s}$ for both the $K_{l 3}$ and hyperon decays. In our approach we find $f_{1} / f_{1}^{S U(3)}<1$ since the wave function overlap is smaller for $\Delta m \neq 0$.

In order to determine the Kobayashi-Maskawa-Cabibbo matrix element $V_{u s}$ we can fit the hyperon decay rate and asymmetries within the Cabibbo model using the $f_{1}$ and $g_{1}$ from Tables VII and VIII, and using the dipole masses from Table VI. We get a value similar to Ref. [30]

$$
V_{u s}=0.225 \pm 0.003[13]
$$

This has to be compared to the value from $K_{e 3}$ which is $0.2196 \pm 0.0023$ [31]. A discussion about this discrepancy can be found in Ref. [30]. Note that the matrix element $V_{u s}$ is a crucial input for the determination of all parameters of the CKM matrix in the framework proposed in Ref. 32]. 


\section{CONCLUSIONS}

In this paper we have analyzed in detail the semileptonic beta decay of the nucleons and hyperons within a relativistic constituent quark model. All parameters of the model have previously been determined by a fit to the magnetic moments of the baryon octet. We see no evidence for configuration mixing. The momentum dependence of the form factors has been calculated and we find some deviation from popular parameterizations. The SU(3) symmetry breaking for the vector and axial form factors is determined. We find that the symmetry breaking for $g_{1}(\Lambda \rightarrow p)$ is of second order. Our value for $V_{u s}$ is somehow larger than the $K_{e 3}$ one in agreement with other studies [29, 30]. We conclude that our relativistic constituent quark model does a good job in analyzing the electroweak properties of the baryon octet.

\section{ACKNOWLEDGMENTS}

It is a pleasure to thank S. J. Brodsky and F. Coester for helpful discussions. This work was supported in part by the Schweizerischer Nationalfonds and in part by the Department of Energy, contract DE-AC03-76SF00515. 


\section{REFERENCES}

[1] Review of Particle Properties, Phys. Rev. D 50, Part 1 (1994).

[2] F. Schlumpf, Phys. Rev. D 47, 4114 (1993); ERRATUM, 49, 6246 (1994).

[3] Z. Dziembowski, C. J. Martoff, and P. Zyla, Report No. PRINT-94-0047 (TEMPLE) (1994).

[4] S. J. Brodsky and F. Schlumpf, Phys. Lett. B 329, 111 (1994).

[5] F. Schlumpf, J. Phys. G 20, 237 (1994).

[6] A. Garcia and P. Kielanowski, The Beta Decay of Hyperons, edited by A. Bohm, Lecture Notes in Physics Vol. 222, (Springer-Verlag, Berlin, 1985).

[7] A. Garcia, Phys. Rev. D 25, 1348 (1982).

[8] J.-M. Gaillard and G. Sauvage, Annu. Rev. Nucl. Part. Sci. 34, 351 (1984).

[9] E. Wigner, Ann. Math. 40, 149 (1939); H. J. Melosh, Phys. Rev. D 9, 1095 (1974); L. A. Kondratyuk and M. V. Terent'ev, Yad. Fiz. 31, 1087 (1980) [Sov. J. Nucl. Phys. 31, 561 (1980)].

[10] F. Coester and W. N. Polyzou, Phys. Rev. D 26, 1349 (1982); P. L. Chung, F. Coester, B. D. Keister and W. N. Polyzou, Phys. Rev. C 37, 2000 (1988); H. Leutwyler and J. Stern, Annals Phys. 112, 94 (1978).

[11] F. Schlumpf, Phys. Rev. D 48, 4478 (1993).

[12] M. Ademollo and K. Gatto, Phys. Lett. 13, 264 (1964).

[13] F. Schlumpf, PhD thesis Zurich (1992), hep-ph-9211255.

[14] G. Karl, Phys. Lett. B 328, 149 (1994).

[15] M. Bourquin et al., Z. Phys. C 12, 307 (1982).

[16] The bag model calculation [23] gives similar results: $g_{1} / f_{1}\left(\Lambda \rightarrow p e^{-} \bar{\nu}_{e}\right)=0.84$, and $g_{1} / f_{1}\left(\Sigma^{-} \rightarrow n e^{-} \bar{\nu}_{e}\right)=-0.28$.

[17] H. J. Lipkin, Report No. WIS-92-105 (1992), hep-ph-9212316.

[18] D. Jensen et al., in Proceedings of HEP83, Brighton, England, edited by J. Guy and C. Costain (Rutherford Appleton Laboratory, Oxfordshire, UK, 1983), p. 255.

[19] S. Y. Hsueh et al., Phys. Rev. D 38, 2056 (1988).

[20] N. Isgur, G. Karl, and R. Konjuk, Phys. Rev. D 25, 2394 (1982).

[21] S. Capstick and N. Isgur, Phys. Rev. D 34, 2809 (1986).

[22] J. Dworkin et al., Phys. Rev. D 41, 780 (1990).

[23] J. F. Donoghue and B. R. Holstein, Phys. Rev. D 25, 206 (1982); J. F. Donoghue, B. R. Holstein and S. W. Klimt, Phys. Rev. D 35, 934 (1987).

[24] S. V. Belikov et al., Z. Phys. A 320, 625 (1985).

[25] J. Brunner et al., Z. Phys. C 45, 551 (1990).

[26] M. Roos, Phys. Lett. B 246, 179 (1990).

[27] P. G. Ratcliffe, Phys. Lett. B 242, 271 (1990).

[28] P. M. Gensini, Nuovo Cim. A 103, 303 (1990).

[29] P. M. Gensini and G. Violini, $\pi N$ Newsletter 9, 80 (1993).

[30] A. Garcia, R. Huerta and P. Kielanowski, Phys. Rev. D 45, 879 (1992).

[31] H. Leutwyler and M. Roos, Z. Phys. C 25, 91 (1984).

[32] A. J. Buras, Phys. Lett. B 333, 476 (1994).

[33] A. Krause, Helv. Phys. Acta 63, 3 (1990).

[34] J. Anderson and M. A. Luty, Phys. Rev. D 47, 4975 (1993). 


\section{TABLES}

TABLE I. Parameters in Eq. (3.1).

\begin{tabular}{cccc}
\hline \hline Reaction & $A\left(f_{1}\right)$ & $A\left(f_{2}\right)$ & $A\left(g_{1}\right)$ \\
\hline$n p$ & 1 & $\left(2 A_{2}-5 A_{3}\right) / 3$ & $\frac{5}{3}$ \\
$\Sigma^{+} \Lambda$ & 0 & $\left(A_{2}+A_{1}-2 A_{3}\right) / \sqrt{6}$ & $\sqrt{\frac{2}{3}}$ \\
$\Sigma^{-} \Lambda$ & 0 & $\left(A_{2}+A_{1}-2 A_{3}\right) / \sqrt{6}$ & $\sqrt{\frac{2}{3}}$ \\
$\Sigma^{-} \Sigma^{0}$ & $\sqrt{2}$ & $-\left(4 A_{3}+A_{2}+A_{1}\right) /(3 \sqrt{2})$ & $\frac{2 \sqrt{2}}{3}$ \\
$\Sigma^{0} \Sigma^{+}$ & $-\sqrt{2}$ & $\left(4 A_{3}+A_{2}+A_{1}\right) /(3 \sqrt{2})$ & $-\frac{2 \sqrt{2}}{3}$ \\
$\Xi^{-} \Xi^{0}$ & -1 & $\left(2 A_{2}+2 A_{1}-A_{3}\right) / 3$ & $\frac{1}{3}$ \\
\hline \hline
\end{tabular}

TABLE II. Parameters in Eq. (3.3).

\begin{tabular}{ccccccc}
\hline \hline Reaction & $B_{1}$ & $B_{2}$ & $B_{3}$ & $B_{4}$ & $B_{5}$ & $B_{6}$ \\
\hline$\Lambda p$ & $-\sqrt{\frac{3}{2}}$ & $-\sqrt{\frac{3}{2}}$ & 0 & $-\sqrt{\frac{3}{2}}$ & 0 & 0 \\
$\Sigma^{0} p$ & $-\frac{1}{\sqrt{2}}$ & $\frac{1}{3 \sqrt{2}}$ & $\frac{\sqrt{2}}{3}$ & $\frac{1}{3 \sqrt{2}}$ & $\frac{4 \sqrt{2}}{3}$ & $\frac{\sqrt{2}}{3}$ \\
$\Sigma^{-} n$ & -1 & $\frac{1}{3}$ & $\frac{2}{3}$ & $\frac{1}{3}$ & $\frac{8}{3}$ & $\frac{2}{3}$ \\
$\Xi^{-} \Lambda$ & $\sqrt{\frac{3}{2}}$ & $\frac{1}{\sqrt{6}}$ & $-\frac{1}{\sqrt{6}}$ & $\frac{1}{\sqrt{6}}$ & $-2 \sqrt{\frac{2}{3}}$ & $-\frac{1}{6}$ \\
$\Xi^{-} \Sigma^{0}$ & $\frac{1}{\sqrt{2}}$ & $\frac{5}{3 \sqrt{2}}$ & $\frac{1}{3 \sqrt{2}}$ & $\frac{5}{3 \sqrt{2}}$ & $\frac{4}{3 \sqrt{2}}$ & $\frac{\sqrt{2}}{6}$ \\
$\Xi^{0} \Sigma^{+}$ & 1 & $\frac{5}{3}$ & $\frac{1}{3}$ & $\frac{5}{3}$ & $\frac{4}{3}$ & $\frac{1}{3}$ \\
\hline \hline
\end{tabular}


TABLE III. Results for $\Delta S=0$ weak beta decay. Experimental data are from PDG 1 .

\begin{tabular}{|c|c|c|c|c|c|c|c|}
\hline & & $n p$ & $\Sigma^{+} \Lambda$ & $\Sigma^{-} \Lambda$ & $\Sigma^{-} \Sigma^{0}$ & $\Sigma^{0} \Sigma^{+}$ & $\Xi^{-} \Xi^{0}$ \\
\hline \multirow[t]{3}{*}{$f_{1}$} & $f_{1}(0)$ & 1.00 & 0 & 0 & 1.41 & -1.41 & -1.00 \\
\hline & $\Lambda_{1}(\mathrm{GeV})$ & 0.69 & $-0.32^{\mathrm{a}}$ & $-0.32^{\mathrm{a}}$ & 0.60 & 0.60 & 0.56 \\
\hline & $\Lambda_{2}(\mathrm{GeV})$ & 0.96 & $-1.72^{\mathrm{a}}$ & $-1.72^{\mathrm{a}}$ & 0.81 & 0.81 & 0.71 \\
\hline \multirow[t]{3}{*}{$g_{1}$} & $g_{1}(0)$ & 1.25 & 0.60 & 0.60 & 0.69 & -0.69 & 0.24 \\
\hline & $\Lambda_{1}(\mathrm{GeV})$ & 0.76 & 0.77 & 0.77 & 0.77 & 0.77 & 0.76 \\
\hline & $\Lambda_{2}(\mathrm{GeV})$ & 1.04 & 1.05 & 1.05 & 1.04 & 1.04 & 1.04 \\
\hline \multirow[t]{3}{*}{$g_{1} / f_{1}$} & Theor. & 1.252 & $0.736^{\mathrm{b}}$ & $0.736^{\mathrm{b}}$ & 0.491 & 0.491 & -0.244 \\
\hline & Expt. & 1.2573 & $0.742^{\mathrm{b}}$ & - & - & - & $<2 \times 10^{3}$ \\
\hline & & \pm 0.0028 & \pm 0.018 & & & & \\
\hline \multirow[t]{2}{*}{$\frac{f_{2}}{M}\left(\mathrm{GeV}^{-1}\right)$} & Theor. & 1.81 & 1.04 & 1.04 & 0.76 & -0.76 & 0.73 \\
\hline & CVC & 1.85 & 1.17 & 1.17 & 0.60 & -0.60 & 1.00 \\
\hline$\frac{g_{2}}{M}\left(\mathrm{GeV}^{-1}\right)$ & & 0 & 0 & 0 & 0 & 0 & 0 \\
\hline Rate $\left(10^{6} s^{-1}\right)$ & Theor. & $1.152 \times 10^{-9}$ & 0.24 & 0.389 & $1.47^{\mathrm{c}}$ & $3.65^{\mathrm{d}}$ & $1.55^{\mathrm{c}}$ \\
\hline \multirow[t]{2}{*}{$e$-mode } & Expt. & $1.127 \times 10^{-9}$ & 0.25 & 0.387 & - & - & - \\
\hline & & \pm 0.003 & \pm 0.06 & \pm 0.018 & & & \\
\hline \multirow[t]{3}{*}{$\alpha_{e \nu}$} & Theor. & -0.101 & -0.404 & -0.412 & 0.436 & 0.438 & 0.793 \\
\hline & Expt. & -0.102 & -0.35 & -0.404 & & & \\
\hline & & \pm 0.005 & \pm 0.15 & \pm 0.044 & & & \\
\hline \multirow[t]{3}{*}{$\alpha_{e}$} & Theor. & -0.112 & -0.701 & -0.704 & 0.287 & 0.288 & -0.514 \\
\hline & Expt. & -0.1127 & & & & & \\
\hline & & \pm 0.0011 & & & & & \\
\hline \multirow[t]{3}{*}{$\alpha_{\nu}$} & Theor. & 0.989 & 0.647 & 0.645 & 0.850 & 0.850 & -0.314 \\
\hline & Expt. & 0.997 & & & & & \\
\hline & & \pm 0.028 & & & & & \\
\hline$\alpha_{B}$ & $\begin{array}{l}\text { Theor. } \\
\text { Expt. }\end{array}$ & -0.548 & 0.070 & 0.077 & -0.710 & -0.711 & 0.518 \\
\hline
\end{tabular}

anstead of $\Lambda_{i}$ we list $f_{1}^{(i)}$.

${ }^{\mathrm{b}}$ Instead of $g_{1} / f_{1}$ we list $\sqrt{3 / 2} g_{1}$.

${ }^{\mathrm{c}} \times 10^{-6}$.

$\mathrm{d} \times 10^{-8}$. 
TABLE IV. Results for $\Delta S=1$ weak beta decay. Experimental data are from PDG [1].

\begin{tabular}{|c|c|c|c|c|c|c|c|}
\hline & & $\Lambda p$ & $\Sigma^{0} p$ & $\Sigma^{-} n$ & $\Xi^{-} \Lambda$ & $\Xi^{-} \Sigma^{0}$ & $\Xi^{0} \Sigma^{+}$ \\
\hline \multirow[t]{3}{*}{$f_{1}$} & $f_{1}(0)$ & -1.19 & -0.69 & -0.97 & 1.19 & 0.69 & 0.98 \\
\hline & $\Lambda_{1}(\mathrm{GeV})$ & 0.71 & 0.64 & 0.64 & 0.68 & 0.75 & 0.75 \\
\hline & $\Lambda_{2}(\mathrm{GeV})$ & 0.98 & 0.84 & 0.90 & 0.89 & 1.05 & 1.05 \\
\hline \multirow[t]{3}{*}{$g_{1}$} & $g_{1}(0)$ & -0.99 & 0.19 & 0.27 & 0.33 & 0.94 & 1.33 \\
\hline & $\Lambda_{1}(\mathrm{GeV})$ & 0.81 & 0.83 & 0.83 & 0.81 & 0.81 & 0.81 \\
\hline & $\Lambda_{2}(\mathrm{GeV})$ & 1.12 & 1.16 & 1.16 & 1.10 & 1.12 & 1.12 \\
\hline \multirow[t]{3}{*}{$g_{1} / f_{1}$} & Theor. & 0.826 & -0.275 & -0.275 & 0.272 & 1.362 & 1.362 \\
\hline & Expt. & 0.718 & - & -0.340 & 0.25 & 1.287 & $<2.93$ \\
\hline & & \pm 0.015 & & \pm 0.017 & \pm 0.05 & \pm 0.158 & \\
\hline \multirow[t]{2}{*}{$\frac{f_{2}}{M}\left(\mathrm{GeV}^{-1}\right)$} & Theor. & -0.85 & 0.44 & 0.62 & 0.070 & 0.98 & 1.38 \\
\hline & CVC & -1.19 & - & 1.12 & -0.080 & 1.38 & 1.95 \\
\hline$\frac{g_{2}}{M}\left(\mathrm{GeV}^{-1}\right)$ & & -0.025 & 0.0043 & 0.0061 & $-^{\mathrm{a}}$ & $-^{\mathrm{a}}$ & $-^{\mathrm{a}}$ \\
\hline Rate $\left(10^{6} s^{-1}\right)$ & Theor. & 3.51 & 2.72 & 5.74 & 2.96 & 0.549 & 0.942 \\
\hline \multirow[t]{2}{*}{$e$-mode } & Expt. & 3.170 & - & 6.88 & 3.36 & 0.53 & - \\
\hline & & \pm 0.058 & & \pm 0.26 & \pm 0.19 & \pm 0.10 & \\
\hline \multirow{3}{*}{$\begin{array}{c}\text { Rate }\left(10^{6} s^{-1}\right) \\
\mu \text {-mode }\end{array}$} & Theor. & 0.58 & 1.18 & 2.54 & 0.80 & $7.47 \times 10^{-3}$ & $7.74 \times 10^{-3}$ \\
\hline & Expt. & 0.60 & - & 3.04 & 2.1 & - & - \\
\hline & & \pm 0.13 & & \pm 0.27 & \pm 2.1 & & \\
\hline \multirow[t]{3}{*}{$\alpha_{e \nu}$} & Theor. & -0.100 & 0.443 & 0.437 & 0.531 & -0.252 & -0.248 \\
\hline & Expt. & -0.019 & & 0.279 & 0.53 & & \\
\hline & & \pm 0.013 & & \pm 0.026 & \pm 0.1 & & \\
\hline \multirow[t]{3}{*}{$\alpha_{e}$} & Theor. & -0.021 & -0.536 & -0.537 & 0.236 & -0.226 & -0.223 \\
\hline & Expt. & 0.125 & & $-0.519^{\mathrm{b}}$ & & & \\
\hline & & \pm 0.066 & & \pm 0.104 & & & \\
\hline \multirow[t]{3}{*}{$\alpha_{\nu}$} & Theor. & 0.992 & -0.318 & -0.318 & 0.592 & 0.973 & 0.973 \\
\hline & Expt. & 0.821 & & $-0.230^{\mathrm{b}}$ & & & \\
\hline & & \pm 0.066 & & \pm 0.061 & & & \\
\hline \multirow[t]{3}{*}{$\alpha_{B}$} & Theor. & -0.582 & 0.568 & 0.569 & -0.519 & -0.437 & -0.439 \\
\hline & Expt. & -0.508 & & $0.509^{\mathrm{b}}$ & & & \\
\hline & & \pm 0.065 & & \pm 0.102 & & & \\
\hline
\end{tabular}

a $\frac{g_{2}}{g_{1} M} \simeq 0.023$ since $\frac{g_{2}}{g_{1}} \simeq$ constant.

${ }^{\mathrm{b}}$ From Ref. [19]. 
TABLE V. Parameters for the configuration mixing of the baryon octet given in Eq. (4.7) for two different references.

\begin{tabular}{lccc}
\hline \hline & $\mathrm{A}$ & $\mathrm{B}$ & $\mathrm{C}$ \\
\hline Ref. [20] & 0.93 & -0.29 & -0.23 \\
Ref. [21] & 0.90 & -0.34 & -0.27 \\
\hline \hline
\end{tabular}

TABLE VI. The parameters $M_{V}$ and $M_{A}$ for various models in units of GeV.

\begin{tabular}{lcccccccc}
\hline \hline & \multicolumn{2}{c}{ This work } & \multicolumn{2}{c}{ Gaillard et al. [8] } & \multicolumn{2}{c}{ Garcia et al. [6] } & \multicolumn{2}{c}{ Gensini [28] } \\
& $M_{V}$ & $M_{A}$ & $M_{V}$ & $M_{A}$ & $M_{V}$ & $M_{A}$ & $M_{V}$ & $M_{A}$ \\
\hline$n p$ & 0.96 & 1.04 & 0.84 & 1.08 & 0.84 & 0.96 & 0.84 & 1.08 \\
$\Sigma \Lambda$ & - & 1.05 & - & 1.08 & - & 0.96 & - & 1.08 \\
$\Sigma \Sigma$ & 0.81 & 1.04 & 0.84 & 1.08 & 0.84 & 0.96 & 0.84 & 1.08 \\
$\Xi \Xi$ & 0.71 & 1.04 & 0.84 & 1.08 & 0.84 & 0.96 & 0.84 & 1.08 \\
$\Lambda p$ & 0.98 & 1.12 & 0.98 & 1.25 & 0.97 & 1.11 & 0.94 & 1.16 \\
$\Sigma p$ & 0.84 & 1.16 & 0.98 & 1.25 & 0.97 & 1.11 & 0.94 & 1.16 \\
$\Sigma n$ & 0.90 & 1.16 & 0.98 & 1.25 & 0.97 & 1.11 & 0.94 & 1.16 \\
$\Xi \Lambda$ & 0.89 & 1.10 & 0.98 & 1.25 & 0.97 & 1.11 & 0.94 & 1.16 \\
$\Xi \Sigma$ & 1.05 & 1.12 & 0.98 & 1.25 & 0.97 & 1.11 & 0.94 & 1.16 \\
\hline \hline
\end{tabular}

TABLE VII. Symmetry breaking for $f_{1}$. The ratio $f_{1} / f_{1}^{\mathrm{SU}(3)}$ is shown.

\begin{tabular}{ccccc}
\hline \hline & This work & Donoghue [23] & Krause [33] & A\&L [34] \\
\hline$\Delta S=0$ & 1.000 & 1.000 & 1.000 & 1.000 \\
$\Lambda p$ & 0.976 & 0.987 & 0.943 & 1.024 \\
$\Sigma p$ & 0.975 & 0.987 & - & - \\
$\Sigma n$ & 0.975 & 0.987 & 0.987 & 1.100 \\
$\Xi \Lambda$ & 0.976 & 0.987 & 0.957 & 1.059 \\
$\Xi \Sigma$ & 0.976 & 0.987 & 0.943 & 1.011 \\
\hline \hline
\end{tabular}


TABLE VIII. Symmetry breaking for $g_{1}$. The ratio $g_{1} / g_{1}^{\mathrm{SU}(3)}$ is shown.

\begin{tabular}{lcc}
\hline \hline & This work & Donoghue [23] \\
\hline$n p$ & 1.000 & 1.000 \\
$\Sigma \Lambda$ & 0.981 & $0.9383 / 0.9390$ \\
$\Sigma \Sigma$ & 0.982 & - \\
$\Xi \Xi$ & 0.977 & - \\
$\Lambda p$ & 1.072 & 1.050 \\
$\Sigma p$ & 1.051 & - \\
$\Sigma n$ & 1.056 & 1.040 \\
$\Xi \Lambda$ & 1.072 & 1.003 \\
$\Xi \Sigma$ & 1.061 & 0.9954 \\
\hline \hline
\end{tabular}

